\title{
Business analytics modeling in Information Automation
}

\author{
MuJun Wan \\ Department of information technology, ZhongShan Torch Polytechnic, \\ Zhongshan GuangDong, China \\ 42397201@qq.com
}

\begin{abstract}
Keywords: business, model, automation, intelligence.
Abstract. Business intelligence (BI) refers to a management process whereby a company can more efficiently execute its business strategies and track its improvement processes. This paper presents that BI has been referred to as an attitude that involves: Understanding the strategic value of data captured from transactions across the enterprise, The organization, standardization and transformation of that data into information. We design a new business intelligence model to reach across all domains and allows business thinkers to ask questions that were impossible to answer prior to the arrival of powerful hardware and analytics.
\end{abstract}

\section{Introduction}

The computerization of nearly all business transactions has led to a virtual avalanche of data. This data can be a rich source of business information. Unfortunately, the data is often contained in information silos that are not linked or integrated. BI has developed as a tool to transform this deluge of date into useful, organized, integrated information that can be searched and analyzed across all the domains existing in a global enterprise. More importantly, proper application of BI can lead to internal efficiency, increased revenue, decreased cost, improved business relationships, and a deeper understanding of the enterprise and its environment, resulting in a sustainable competitive advantage.

It is difficult to calculate the BI marketplace because BI refers to many products which may or may not be integrated with other systems. Online analytic processing or OLAP is a business intelligence function. The OLAP market serves as a proxy for the general BI market and demonstrates the high demand for business analysis. Despite the "boom and bust" cycles seen in most other IT areas, BI has seen steady growth and the recent consolidation in the BI vendor space speaks to the importance and maturity of this market. As quoted from the OLAP report: "Readers should not attempt to view the OLAP market in isolation. It overlaps many other 'markets', including analytical applications, performance management, data warehousing, CRM, EIS, decision support, query \& reporting, enterprise reporting and even databases, so it is misleading to try to aggregate the figures of several of these indistinct sectors to calculate a total 'business intelligence' market size." Nonetheless, the OLAP report predicts a $\$ 5$ billion market by 2014 .

BI offers tools to manage the impossibly large amount of data available to a business. This includes data previously isolated in silos across the enterprise. In addition, BI tools can now allow integration with data from partners and other sources outside the corporate boundary. When properly implemented and understood as a corporate philosophy, BI enforces data standardization and integration, offering a view of the business that is panoramic and that was previously unobtainable. With such a view, new insights into operations and performance can emerge and result in superior execution.

\section{BI system analysis}

The need for BI has grown concurrently with the data available for analysis. The persistence of Moore's law and it's corollary for data storage allow for an ever improving cost efficiency curve for computing and data storage in the enterprise. Born out of this marriage of power and storage is a colossal data 'monster'. The amount of data collected by existing and planned transaction systems continues to increase at an astounding rate. External data sources are also growing rapidly in number 
and raw content. Gartner believes that by 2013 , enterprises will need to deal with thirty times more data than in 2012.

The current drive for globalization, increasing competition and intense performance pressure all mandate improved, integrated information systems. Data integrity, integration and access are more important than ever to smart managers. Without BI initiatives to create a data gathering and analysis architecture and the corporate will to create a parallel BI culture amongst decision makers the data harvest will rot in the barn or lie fallow in the field. Successfully managing such data stores can be the determining factor in obtaining market share and the difference between the success and failure of the corporation. While BI is a maturing discipline, integration remains the key to success. Earlier BI projects tended to focus on specific business functions, leading to isolated projects with well known acronyms like CRM (customer relationship management), CPM (corporate performance management), and ERP (enterprise resource planning). This can lead to the creation of new silos of information. BI is offered as the tool to integrate all information of importance, presenting a 'single version of the truth' to decision makers 'at the speed of thought'. This is illustrated in the following diagram :

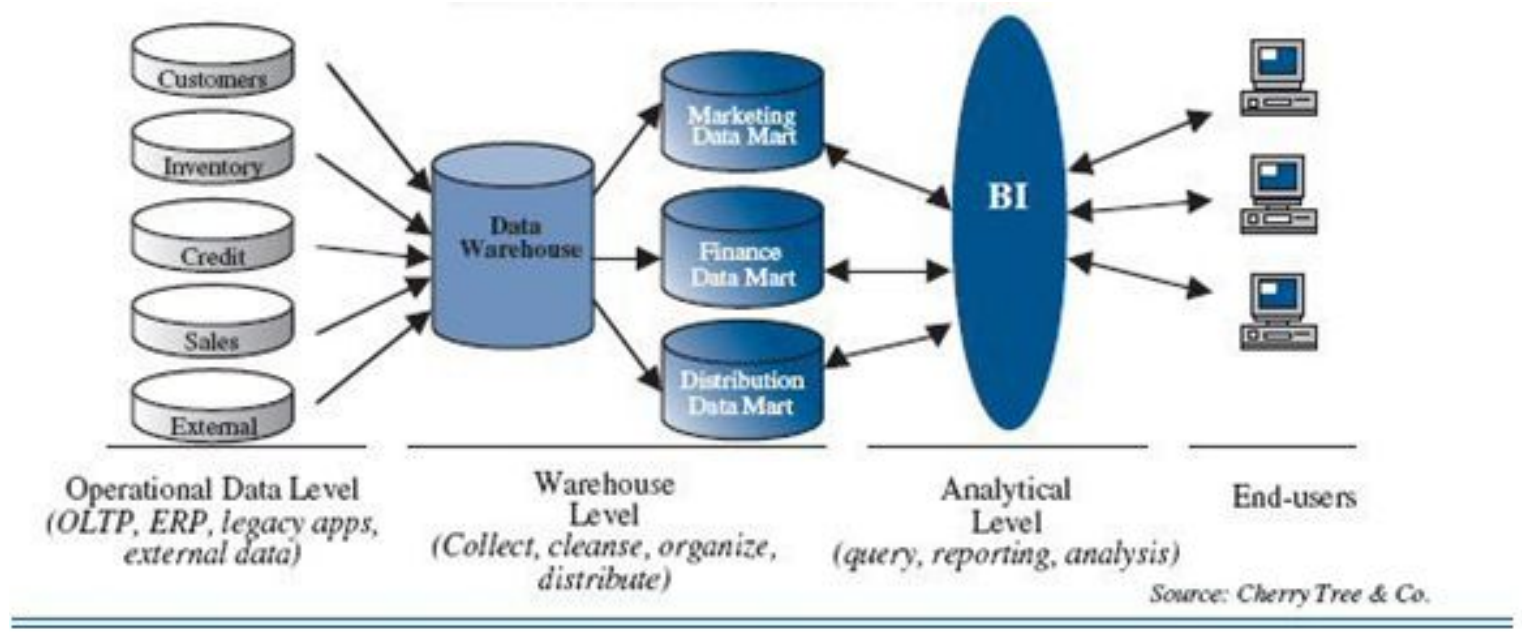

Figure 1 Business intelligence flow chart

As shown in Fig1, Data from disparate sources is extracted, transformed and loaded into a central data warehouse where it is at the beck and call of users via a friendly intermediary BI software layer that provides ad-hoc queries. Mangers across the enterprise (in far flung locations) access the same store of uniform, transformed data, enabling better insight and action.

Finally, managerial accounting has not kept pace with the paradigm shift in data acquisition and analysis. "Today most observers agree that management accounting information derived from financial accounting systems is obsolete .... Basically, the current system generates incomplete information in which the organization's managers have no confidence. And yet the managers use that information because they have nothing better. The organization also lacks information for systematically, consistently, and routinely performing fundamental operations management trade-offs among asset levels, costs, process times, quality service, outputs and backlogs." The promise of BI - instantaneous analytical access to accumulated global enterprise data resulting in faster, better decisions - as envisioned by managerial accounting, is illustrated in the next figures, which depict a shift away from the constraints of traditional financial accounting systems to a modern Management Accounting Information Framework based on integration of data sources and the ability to specify appropriate cost models as needed.

\section{System design}

SiChuan Province Health care continues to face major challenges including declining reimbursement, increased acuity levels (sicker patients), ever increasing investments in medical technology, alienation of the medical staff (via competing surgical centers), nursing shortages, bizarre business rules and increasing scrutiny of clinical outcomes. This last point is most important. 
At a time of intense financial pressure and resource shortages, where a tradition of perverse incentives emphasizes pay for production but not for quality, Medicare has begun to suggest that payment be linked to performance on a selected subset of clinical outcomes. This is in part due to the fervor created by the widely quoted 1999 Institute of Medicine's report on medical error "To Err is Human" , in which 98,000 patient deaths per year were attributed to medical error.

This encouraged large corporations, the largest purchasers of insurance, to band together to form the Leapfrog Group, to pressure health care systems to adapt a quality culture enabled by clinical information systems. This is a paradigm shift of historical significance. Health care IS has traditionally focused on financial reporting, the claims data that flows as the monetary lifeblood of the organization. Historically, clinical IS has emphasized only static results reporting and clinical IS decision support was essentially nonexistent. In the absence of clinical IS then, how can quality be improved? As we move through the decade it will take to implement the electronic health record (EHR) and its constituent components - computerized physician order entry (CPOE), barcode medication administration (BMA) and clinical decision support (CDS) .

In the health care sector business intelligence can improve patient outcomes, forecast revenue, suggest staffing levels and anticipate problems with quality reporting that put the institution at risk. Comparing data both internally across regions and externally via a commercial data warehouse can motivate and empower decision makers, leading to improvements in patient care and financial performance. In the following example, Indeed, given the intense financial, utilization and regulatory pressures on CDOs, BI may be the only way to survive the next decade.

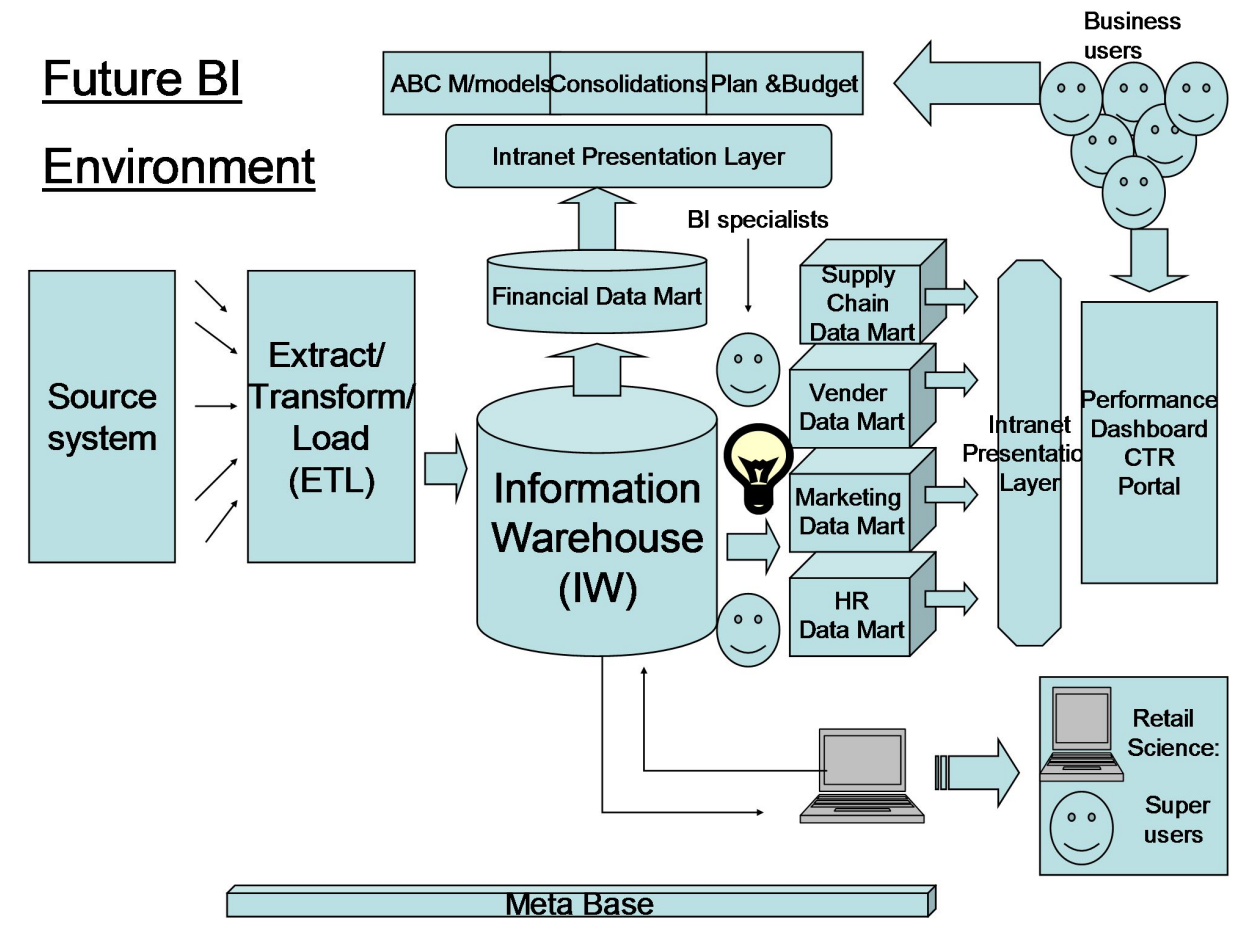

Figure 2 the improved BI system architecture

We present an improved model in Fig2, which illustrates the future of BI for CTC. Independent data sources have been incorporated into a single BI system, within a global data model. All data will loaded into the corporate IW via ETL. The IW will feed the financial data mart for enterprise performance management, so business users will be able to conduct planning and budgeting functions. On the right side of the IW, BI specialists will assist in organizing data marts into four categories: Supply Chain, Vender, Marketing, and HR Data Mart. In the Performance Dashboard business users will be able to get instant end-user data. In a distinct change from the prior BI model, business users won't need to ask for business support, reporting, or ac hoc analysis help. Historical statistical information goes directly to super users via the retail science application, noted at the bottom of the IW in the diagram. Super users will use this information to forecast the future market trends. 
BI vision at CTC was to provide "the right information for the right decision at the right time, enabling proactive, accurate business decisions."i The BI program goals were: To develop an enterprise philosophy that held the real value of an optimized BI environment. To encourage a culture valuing high data quality. To foster and enable the CTR business strategies and IT strategies. To develop BI efficiency through cross-functional synergies within business intelligence and data management. To define and incrementally implement the technology changes required to enable and sustain the business BI goals and objectives.

An engine, system and method for providing cloud-based business intelligence data, responsively to received company information, including a local front end comprising a graphical user interface capable of locally querying a user for an electronic location of the received company information, and ones of the business intelligence data to be displayed on the graphical user interface upon upload of the received company information from the electronic location, and further including an at least partially remote back end, comprising an accessing engine capable of accessing the electronic location for uploading to the cloud of only ones of the received company information indicative of the ones of the business data intelligence to be displayed, and a charging engine for charging an account of a user of the front end $\$ 1$ per the ones of the business intelligence data to be displayed.

\section{Conclusions}

In this paper, we have presented an improved, effective and efficient method for dealing with large data streams. This paper presents that BI has been referred to as an attitude that involves: Understanding the strategic value of data captured from transactions across the enterprise, The organization, standardization and transformation of that data into information. We design a new business intelligence model to reach across all domains and allows business thinkers to ask questions that were impossible to answer prior to the arrival of powerful hardware and analytics. A big data analytics system obtains a plurality of manufacturing parameters associated with a manufacturing facility. The big data analytics system identifies first real-time data from a plurality of data sources to store in memory-resident storage based on the plurality of manufacturing parameters.

\section{References}

[1]ElizabethVitt, Michael Luckevich, Stacia Misner. "Business Intelligence: Better Decisions Faster", p16. Redmond, Washington: Microsoft Press, 2002.

[2] Larissa T. Moss, Shaku Atre. "Business Intelligence Roadmap. The Complete Project Lifecycle for Decision-Support Applications”, Page 5. Boston, MA: Addison-Wesley 2013

[3] Williams, Steve. Delivering Strategic Business Value. Strategic Finance; Aug 2014; 86, 2, pp. $42-48$

[4] Raymond, Louis. Globalization, the knowledge economy, and competitiveness: A business intelligence framework for the development of SMES. Journal of American Academy of Business, Cambridge; Sep 2003; 3, 1/2; p. 260

[5] Holbrook, Kevin. Adding Value with analytics. Strategic Finance; Nov 2004; 86, 5; p 41 